\title{
GAINS: neutron inelastic cross section measurements of interest for applica- tions and reaction studies
}

\author{
A. Negret ${ }^{1, *}, C$ Borcea $^{1}, M$. Boromiza $^{1}, P h$. Dessagne $^{2}, G r$. Henning $^{2}, M$. Kerveno $^{2}, M$. Nyman $^{3}, A$. Olacel $^{1}$, and $A . J . M$. \\ Plompen $^{3}$ \\ ${ }^{1}$ Horia Hulubei National Institute for Physics and Nuclear Engineering, 077125 Bucharest-Măgurele, Romania \\ ${ }^{2}$ Université de Strasbourg, CNRS, IPHC UMR7178,F-67000 Strasbourg, France \\ ${ }^{3}$ European Commission, Joint Research Centre - Geel, B-2440 Geel, Belgium
}

\begin{abstract}
The paper represents an overview of the measurements performed using GAINS at GELINA (JRCGeel, Belgium). While undergoing continuous upgrades, the setup produced highly precise cross sections. Our measurements are primarily driven by technological needs with an emphasis on structural materials used in the development of nuclear facilities. However, most cases offered the opportunity to investigate various reaction mechanism and/or nuclear structure issues. We concentrate on several specific experiments describing the particular difficulties we met and the solutions we adopted to infer reliable data and to draw significant conclusions.
\end{abstract}

\section{Introduction}

Despite the fact that the components of the atomic nucleus were discovered almost a century ago, a complete understanding of the nuclear structure still eludes the possibilities of the modern science. Two ingredients are currently missing from the image we have about the nucleus: a complete mathematical form of the nuclear force and a detailed framework able to describe the mesoscopic systems. Despite these important difficulties the nuclear physics was able to understand an impressive amount of experimental observations. Even more important, it was able to develop a significant number of applications with considerable impact on the society: energy production, medicine, safety and security, industrial imaging, etc. However, most of these applications are possible not necessarily due to a detailed understanding of the nuclear structure but rather due to the important amount of very precise experimental information gathered during the years: this is what we call nuclear data. Indeed, following the extensive experimental programs implemented during the last century both in the field of nuclear structure and of the nuclear reactions, we posses now databases that make possible practically all applications currently available. Further, the development of new applications that may bring benefits to the society depends on the improvement of these databases. Therefore the field of nuclear data represents nowadays a quest for reliability and precision.

In this context we developed the GAINS (Gamma Array for Inelastic Neutron Scattering) setup [1] at the GELINA [2] (Geel Linear Accelerator) neutron source with the definite goal of measuring neutron inelastic cross

*e-mail: alnegret@tandem.nipne.ro sections with low uncertainty while controlling and understanding as well as possible all experimental details in order to gain confidence and reliability. The setup is dedicated to measurements of nuclei that constitute structural materials for present and future facilities. Particularly in the nuclear reactors, inelastic scattering plays a key role during the process of neutron thermalization as it is the main energy loss mechanism. A second setup dedicated to neutron inelastic scattering on actinides, GRAPhEME, is also operational at GELINA; a separate contribution from the current proceedings presents an overview of this setup [3]. The experimental program of both facilities was driven to a large extent by the experimental issues listed in the HPRL (High Priority Request List) database maintained by the Nuclear Energy Agency [4] but also by other scientific cases, as it will be detailed in the present overview.

\section{The setup. Evolution timeline}

The current version of GAINS consists of 12 HPGe detectors centered around the sample under investigations at angles of $110^{\circ}, 125^{\circ}$ and $150^{\circ}$ with regard to the neutron beam provided by GELINA, the pulsed time-of-flight facility operated by the Joint Research Center of the European Commission in Geel, Belgium. The current flight path is $100 \mathrm{~m}$. The neutron beam has a diameter of $6.1 \mathrm{~cm}$, it is pulsed at a repetition rate of $800 \mathrm{~Hz}$ and each pulse has a duration of about 1-2 ns. These characteristics combined with the specific energy resolution of the HPGe detectors allow highly precise cross section measurements using the $\gamma$-spectroscopy and the time-of-flight techniques. The primary experimental results consist of the angle-integrated $\gamma$-production cross sections and, using the level scheme of 
the studied nucleus from an external database, we infer the level and the total inelastic cross sections.

\begin{tabular}{|c|c|}
\hline \multirow[b]{2}{*}{ year } & GAINS Timeline \\
\hline & \\
\hline 2000 & $\begin{array}{l}\text { First attempts to measure }\left(n, n^{\prime} \gamma\right) \text { at GELINA } \\
2 \text { HPGe detectors, flight path }=200 \mathrm{~m}\end{array}$ \\
\hline 2002 & System upgraded to $4 \mathrm{HPGe}$ detectors \\
\hline 2004 & Implementation of the digital DAQ \\
\hline 2006 & $\begin{array}{l}\text { Installation of the first frame, } \\
4 \text { HPGe detectors, }\end{array}$ \\
\hline 2008 & Name of GAINS given to the setup. \\
\hline 2010 & Upgrade to $8 \mathrm{HPGe}$ detectors \\
\hline 2012 & Upgrade to $12 \mathrm{HPGe}$ detectors \\
\hline 2014 & \\
\hline 2016 & $\begin{array}{l}\text { Installation of the second frame, } \\
\text { Dispachment to flight path }=100 \mathrm{~m} \\
\text { Upgrade to } 16 \mathrm{HPGe} \text { detectors }\end{array}$ \\
\hline 2018 & \\
\hline 2020 & Upgrade of the DAQ \\
\hline
\end{tabular}

Figure 1. Evolution of the GAINS array along the time.

But before reaching the current status, the GAIN setup developed through several stages. Already from the beginning the choice of high volume HPGe detectors was triggered by the fact that, in order to measure cross sections on structural materials on a long flight path, a high $\gamma$-detection efficiency was necessary. The long flight path allowed high neutron energy measurements, of the order of $1 \mathrm{keV}$ for $1-\mathrm{MeV}$ neutrons [1].

Figure 1 illustrates very concisely the evolution timeline of the setup from the initial 2 HPGe detectors to the current stage where 12 HPGe detectors are in use. A key upgrade consisted of the implementation of the digital acquisition in 2004-2006 with a dedicated software being finalized in 2008. An upgrade of this DAQ (both of the hardware and of the software) is foreseen for the near future.

We emphasize that, along the timeline displayed in Fig. 1, the experimental program developed in parallel as the setup was continuously exploited both for producing neutron data and for educational purposes, several $\mathrm{PhD}$ students and postdocs being trained during these years at GAINS. We will detail these aspects in the next sections.

\section{Experimental program}

As already mentioned in the introduction, the main purpose of GAINS was to address major chalenges related to the precise measurement of neutron inelastic cross sections on structural materials of importance for the development of nuclear facilities. The Table 1 represents a list of the most important measurements finalized along the years, together with the names of the main investigator (usually a $\mathrm{PhD}$ student or a postdoc) and the corresponding reference. We only list here the experiments were completed and published. There is a natural delay of several years between the date of the actual measurement and the completion of the data analysis and the publication of the corresponding paper or PhD thesis. An interesting exception from this rule was the recent case of the measurement on ${ }^{54} \mathrm{Fe}$ that was performed, analyzed and submitted for publication within one year (2017-2018). The corresponding reference from Tab. 1 but also a separate contribution to the present proceedings [5] offer more details about this result.



Figure 2. Example of $\gamma$-production cross section obtained with GAINS: the $847-\mathrm{keV}$ transition from the ${ }^{56} \mathrm{Fe}(n, n \gamma)$ reaction.

Another specific case is ${ }^{206} \mathrm{~Pb}$ that was measured twice using two different samples, by two main investigators at various moments of the evolution of GAINS. The results, being in perfect agreement, were published together in 2015. A recent measurement offered the opportunity of a second reliability test: the target used for the ${ }^{16} \mathrm{O}$ measurement consisted of a quartz $\left(\mathrm{SiO}_{2}\right)$ plate, thus allowing the measurement of the $\gamma$-production cross sections on ${ }^{28} \mathrm{Si}$ and their comparison with the results published in 2013 using a metallic sample. This inter-comparison, together with the results on ${ }^{16} \mathrm{O}$, are discussed in Ref. [6].

Finally, we note that the measurement of the neutron inelastic cross sections on ${ }^{56} \mathrm{Fe}$ (displayed in Fig. 2) was particularly detailed and carefully performed: aside from the long run using a 3-mm thick target of ${ }^{n a t} \mathrm{Fe}$, we performed various one-week checks varying the target thickness or using other targets $(\mathrm{Ni}, \mathrm{Si})$ to check the background conditions. All details are given in the corresponding reference and up to this date our group did not encountered any issue regarding the results published in 2014.

\section{Particular issues}

We will dedicate this section to several issues encountered during our experiments and to the solutions we used. More details on each specific case can be found in the corresponding publications.

\subsection{The lowest $\gamma$ energy accesible with GAINS: ${ }^{57} \mathrm{Fe}$}

The lowest $\gamma$ energy we tried to measure was the 14.4-keV transition decaying from the first excited state in ${ }^{57} \mathrm{Fe}$. The 
Table 1. List of neutron inelastic cross section measurements performed at GAINS over the years together with the corresponding references.

\begin{tabular}{lll}
\hline \hline Nucleus & Main investigator(s) & Reference \\
\hline${ }^{52} \mathrm{Cr}$ & L.C. Mihăilescu & PhD Thesis, Univ. of Bucharest (2006) \\
& & Nucl. Phys. A786, 1 (2007) \\
& Nucl. Phys. A811, 1 (2008) \\
${ }^{208} \mathrm{~Pb}$ & L.C. Mihăilescu & Nucl. Instrum. Meth. Phys. Res. A672, 82 (2012) \\
${ }^{23} \mathrm{Na}$ & C. Rouki & Phys. Rev. C88, 034604 (2013) \\
${ }^{28} \mathrm{Si}$ & A. Negret & Phys. Rev. C88, 054613 (2013) \\
${ }^{76} \mathrm{Ge}$ & C. Rouki & Phys. Rev. C90, 034602 (2014) \\
${ }^{56} \mathrm{Fe}$ & A. Negret & Phys. Rev. C90, 034603 (2014) \\
${ }^{24} \mathrm{Mg}$ & A. Olacel & Phys. Rev. C91, 064618 (2015) \\
${ }^{206} \mathrm{~Pb}$ & A. Negret \& L.C. Mihăilescu & PhD Thesis, University of Bucharest (2016) \\
${ }^{46-50} \mathrm{Ti}$ & A. Olacel & Phys. Rev. C96, 014621 (2017) \\
& & Phys. Rev. C93, 024610 (2016) \\
${ }^{7} \mathrm{Li}$ & M. Nyman & Phys. Rev. C96, 024620 (2017) \\
${ }^{57} \mathrm{Fe}$ & A. Negret & Eur. Phys. Journal A54 (2018) \\
${ }^{54} \mathrm{Fe}$ & A. Olacel & PhD Thesis, University of Bucharest (2018) \\
${ }^{16} \mathrm{O}$ & M. Boromiza & Article in preparation (2019) \\
& & Article in preparation (2020) \\
${ }^{58,60,61,62,64} \mathrm{Ni}$ & A. Olacel & \\
\hline
\end{tabular}

conversion coefficient of that transition equals 8.56(26) [7] and the 14.4-keV $\gamma$ ray is strongly absorbed in the sample. However, all HPGe detectors of GAINS are equipped with thin entrance windows allowing detection of low-energy $\gamma$ rays. This was indeed proven using a ${ }^{57} \mathrm{Co}$ source that populates, following $\beta$ decay, excited states in ${ }^{57} \mathrm{Fe}$.

Unfortunately, the detection of the $14.4-\mathrm{keV} \gamma$ ray emitted following the inelastic scattering of neutrons on ${ }^{57} \mathrm{Fe}$ was not possible due to the high level of background induced by the neutrons at low $\gamma$ energies that extends at least up to $30-40 \mathrm{keV}$. Consequently, we identified this as a limitation of GAINS: indeed, the elastic scattering of neutrons on the germanium nuclei causes recoil. This energy is deposited in the crystal and collected causing the background increase at small amplitudes.

In order to actually measure the $14.4-\mathrm{keV}$ transition from ${ }^{57} \mathrm{Fe}$ we intend to use the GRAPhEME setup available at GELINA [3] that consist of low-volume HPGe detectors, much less sensitive to low-energy background caused by the elastic scattering of neutrons in the crystals.

\subsection{The highest $\gamma$ energy measured with GAINS: ${ }^{16} \mathrm{O}$}

At the other extreme, the highest $\gamma$ energy measured with GAINS was the 6129.9-keV E3 transition decaying from the second excited state to the ground state of ${ }^{16} \mathrm{O}$. In this case the energy ranges of the HPGe detector preamplifiers was extended by decreasing their gain, on the expense of a significant lost in terms of energy resolution. This allowed, in principle, detection of $\gamma$ rays with energies up to about 10-12 MeV.

The detector efficiencies that are usually determined using a standard ${ }^{152} \mathrm{Eu}$ calibration source were extrapolated to $6129.9-\mathrm{keV}$ using a Monte Carlo simulation. The procedure is not unusual as we routinely use this procedure to account for the $\gamma$ absorption in the sample and to interpolate the efficiencies at the $\gamma$ energies of interest. However, in case of the $6129.9-\mathrm{keV}$ transition we adopted a conservative approach by increasing the uncertainty of our detector efficiencies in order to account for any possible errors caused by the extrapolation procedure.

\subsection{The neutron inelastic cross section resonances: compound nucleus states versus Ericsson fluctuations}

Figure 2 shows the $847-\mathrm{keV} \gamma$-production cross section from the ${ }^{56} \mathrm{Fe}$ reaction reported in 2014 (see Table 1). One of the issues we investigated was related to the nature of the structures observed in the cross section. We emphasize that this type of investigation is possible due to the good time resolution (resulting in good neutron energy resolution) of GAINS.

We compared the level density in the compound nucleus calculated using a standard theoretical approach with the number of resonances per $\mathrm{MeV}$ directly counted from our $\gamma$-production cross sections. As expected, the conclusion depends on the mass number of the investigated nucleus and on the energy: in case of the very light ${ }^{25} \mathrm{Mg}$ compound nucleus [excited in the ${ }^{24} \operatorname{Mg}\left(n, n^{\prime}\right)$ reaction] the theoretical level density was almost equal with the number of resonances observed in the excitation functions for the first $\mathrm{MeV}$ after the threshold. Above this limit the two numbers start to diverge rapidly. In case of the heavier compound nucleus ${ }^{57} \mathrm{Fe}$ [excited in ${ }^{56} \mathrm{Fe}\left(n, n^{\prime}\right)$ ] we noted that already for the first $500 \mathrm{keV}$ after the threshold the theoretical level density is significantly larger than the number of resonances we observe. Consequently we concluded that, even in case of very light nuclei, the structures observed in our experimental $\gamma$-production cross sections correspond to individual levels in the compound nuclei only for a very limited energy range and above this range they are mostly overlaps of such states.

More details about this investigation can be found in the articles dedicated to ${ }^{24} \mathrm{Mg}$ and ${ }^{56} \mathrm{Fe}$ listed in Table 1. 


\subsection{Neutron inelastic scattering as a source of background for neutrinoless double- $\beta$ decay experiments}

One last horizontal investigation we wish to mention exploited several of our experimental datasets to perform an assessment of possible sources of background affecting the experiments attempting to observe neutrinoless double$\beta$ decay. Such experiments are normally conducted in deep underground laboratories where neutrons and particularly their inelastic scattering can produce background that would mimic the signature of neutrinoless double $\beta$ decay events. Neutrons can originate from the interaction of cosmic muons with the environment or from $(\alpha, n)$ reactions triggered following the $\alpha$ decay of the uranium or thorium from the rocks.

The study was presented in Ref. [8]. We identified a 2041-keV transition in ${ }^{206} \mathrm{~Pb}$ and a $2035-\mathrm{keV}$ transition in ${ }^{56} \mathrm{Fe}$ tht could represent sources of background in the attempt to detect the specific signature of the neutrinoless double $\beta$-decay of ${ }^{76} \mathrm{Ge}$.

\section{Conclusions}

The conclusions of this overview are of scientific but also of social nature. First of all, the GAINS setup installed at GELINA produced during the last years highly precise and reliable neutron data of importance for applications but also addressing various basic science issues. Second, the setup represented a very important training ground for several generations of researchers: the second column from Table 1 represents a list of several of these scientists.

Both these aspects will continue to be pursued during the next years. The setup will continue to be developed while new students are expected to get involved in the ongoing experimental program.

The authors thank the team of operators of the GELINA facility for the preparation of the neutron beam and to the technical staff of JRC-Geel for their support during the data taking campaign. This work was partially supported by the European Commission through the ANDES (EURATOM Contract number FP7-249671), EUFRAT (EURATOM Contract number FP7-211499), and CHANDA (EURATOM contract number FP7-605203) projects.

\section{References}

[1] L.C. Mihailescu, L. Olah, C. Borcea, and A.J.M Plompen, Nucl. Instrum. Meth. Phys. Research A531, 375 (2004).

[2] D. Ene, C. Borcea, S. Kopecky, W. Mondelaers, A. Negret, and A.J.M. Plompen, Nucl. Instrum. Meth. Phys. Research A618, 54 (2010).

[3] M. Kerveno et al., Proceedings of the ND2019 conference, Beijing, China (2019).

[4] https://www.oecd-nea.org/dbdata/hprl.

[5] A. Olacel et al., Proceedings of the ND2019 conference, Beijing, China (2019).

[6] M. Boromiza et al., Proceedings of the ND2019 conference, Beijing, China (2019).

[7] M.R. Bhat, Nucl. Data Sheets 85, 415 (1998).

[8] A. Negret, C. Borcea and A.J.M. Plompen, Phys. Rev. C88, 027601 (2013). 